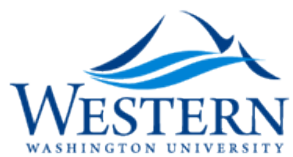

Western Washington University Western CEDAR

2015

\title{
Military Service and Alcohol Use in the United States
}

Jay Teachman

Western Washington University, jay.teachman@wwu.edu

Carter Anderson

Western Washington University

Lucky M. Tedrow

Western Washington University, Lucky.Tedrow@wwu.edu

Follow this and additional works at: https://cedar.wwu.edu/sociology_facpubs

Part of the Sociology Commons

\section{Recommended Citation}

Teachman, Jay; Anderson, Carter; and Tedrow, Lucky M., "Military Service and Alcohol Use in the United States" (2015). Sociology. 14.

https://cedar.wwu.edu/sociology_facpubs/14 
Military Service and Alcohol Use in the United States

\title{
Jay Teachman', Carter Anderson', and Lucky M. Tedrow'
}

\begin{abstract}
It is well known that enlistees and veterans in the United States are more likely to use alcohol than civilians. However, most of this research is potentially biased in that it often does not employ control variables (other than age) and is based on crosssectional data. Much of this research also fails to consider the relationship between military service and alcohol use among women. Using longitudinal data taken from the 1997 National Longitudinal Study of Youth, we investigate the relationship between military service and alcohol consumption employing a fixed-effects approach. We find that military service appears to encourage young men to consume alcohol. It is also the case that the effect of military service is not limited to the time that men spend in the military given that male veterans are also more likely to consume alcohol than are comparable nonveterans. We find, however, that women who serve, both enlistees and veterans, are less likely to drink than their civilian counterparts.
\end{abstract}

\section{Keywords}

military service, alcohol use

Considerable research has documented the fact that alcohol use is positively associated with military service in the United States, at least for men. A recent report by the Institute of Medicine ${ }^{1}$ indicates that over the past thirty years US military personnel

\footnotetext{
'Western Washington University, Bellingham, WA, USA
}

\section{Corresponding Author:}

Jay Teachman, Department of Sociology, Western Washington University, Bellingham, WA 98225, USA. Email: jay.teachman@wwu.edu 
drink more than civilians. Other research suggests that patterns of heavy alcohol use continue after service members leave the military. According to the Substance Abuse and Mental Health Services Administration, ${ }^{2}$ veterans are more likely than nonveterans to drink and drink heavily. At question, however, is the direction of the relationship between military service and alcohol use. Does military service lead to increased use of alcohol? Or, do men and women predisposed to use alcohol choose to enter the military in greater numbers than other individuals? These questions are important because they are tied to policy efforts aimed at reducing the link between military service and use of alcohol. ${ }^{3}$ In this article, we address the issue by using a statistical model that helps minimize the effects of selectivity on longitudinal data taken from the 1997 National Longitudinal Survey of Youth (NLSY-97). We find evidence to support the notion that military service leads to increased levels of alcohol use for men both among individuals currently serving and among veterans. However, the relationship between military service and alcohol is substantially different for women.

\section{Prior Literature}

The prior literature is consistent in finding that military service in the United States and alcohol use are positively related, particularly for men. Using measures such as alcohol use in the past thirty days, binge drinking (five or more drinks on a single day in the past thirty days), or heavy drinking (five or more drinks on a single day for at least five days in the past thirty days), researchers have found that both currently enlisted service members ${ }^{4-6}$ and veterans ${ }^{7-9}$ are more likely to use alcohol. Given the well-known negative health consequences of alcohol use, ${ }^{10}$ these findings are important given the large number of young Americans who enter the military each year (currently about 200,000 young men and women).

Previous research on the topic suffers from several limitations. First, much of the research fails to directly compare service members and veterans to civilians, making it difficult to assess the relative level of alcohol use associated with military service. ${ }^{11-14}$ Second, some of this research also relies on administrative records for treatment of alcohol problems, or samples drawn from respondents seeking health care assistance, failing to measure overall use of alcohol in the military. ${ }^{15-17}$ Third, even if research uses data on civilians to draw conclusions about the relative magnitude of alcohol use linked to military service, the data used are cross-sectional. We are not aware of research examining the relationship between military service and alcohol use that uses longitudinal information on respondents, both civilian and military. Thus, no information is provided about histories or trajectories of alcohol use. This is a serious limitation because questions of causal order are left unanswered making it difficult if not impossible to establish reasonable inferences pertaining to cause and effect.

Moreover, the available research comparing civilians to service members and veterans uses a rather limited set of control variables making it difficult to assess the 
extent to which results are free from problems associated with selectivity into the military as opposed to effects resulting from military service itself. Critical variables that may increase both the likelihood of military service and alcohol use are ignored, as well as factors stemming from military service and experience such as deployments and exposure to combat. There are no results that provide readers with a handle on the likelihood that higher rates of alcohol use among service members and veterans may be due to positive selection into the military by individuals who are more likely to use alcohol, rather than the nature of military service itself.

We address the limitations in prior research in several ways. First, we analyze alcohol consumption using data on both civilians and respondents who have military experience. Second, the data we use are longitudinal, allowing us to take advantage of information about changes in alcohol use over time. In essence, each respondent serves as their own control as they move from being a civilian, to active duty service, to being a veteran. Third, we use an extended set of covariates to better address the problem of selectivity. Although our approach still has limitations, which we discuss later, our results provide a cleaner estimate of the degree to which military service itself affects use of alcohol.

\section{Interpretive Context}

The military in the United States is seen as an institution where heavy drinking is part of its culture. ${ }^{18-20}$ It is also an institution largely comprised of young Americans at those ages where drinking is seen as a rite of passage, ${ }^{21}$ and where peer pressure found in social networks is a prime determinant of alcohol use. ${ }^{22,23}$ In addition to peer influences, alcohol consumption among enlistees is encouraged by price supports in the military. ${ }^{24}$ Alcohol purchased on base is less expensive than alcohol purchased elsewhere. Because of its supportive culture and price supports, we expect that military service leads to increased alcohol consumption.

It is also possible that military service leads to longer term alcohol use after military service ends. For one, alcohol consumption in the military occurs at those ages (late adolescence and early adulthood) at which young persons are developing adult identities. ${ }^{25}$ Alcohol use, in an environment with considerable peer pressure supporting alcohol consumption, may become part of an individual's established sense of self. It is also the case that drinkers can become dependent on alcohol, leading to long-term use. ${ }^{26}$ Alcohol use while serving in the military may therefore translate to habits and patterns of use that extend well beyond time of service. These points lead us to expect that veterans will have higher levels of alcohol abuse than nonveterans.

It may be the case that duration of exposure to military culture with its emphasis on alcohol may also affect use. Longer exposure to peer pressure and alcohol use during a time in the life course when many habits are being formed may lead to an increased likelihood of current and subsequent use. Research has found that greater exposure to peers who use alcohol increases alcohol use. ${ }^{27}$ 
Accordingly, we expect that longer exposure to military culture will be linked to increased use of alcohol.

Some research suggests that gender moderates the relationship between peer groups, alcohol initiation, and alcohol dependence. Prior research suggests that women in the military and women veterans are less likely to use alcohol than their male counterparts. ${ }^{28-30}$ In general, women are less likely to use alcohol than men, and when they do they are less likely to engage in excessive drinking. ${ }^{31}$ Women are also a much smaller percentage of the military population than are men (although their numbers are climbing), and they are less likely to have been exposed to an established a culture of drinking. Moreover, women may be less influenced by the "macho" culture of male drinking in the military. Accordingly, we expect that military service will have less impact on alcohol use among women than among men.

The expectations just formulated all assume that military service in the United States influences alcohol use. An alternative thesis is that alcohol users, or individuals with a propensity to drink alcohol, are drawn to service in the military. Some evidence suggests that individuals who drink heavily are more likely to enlist or be interested in enlisting in the military than individuals who abstain. ${ }^{32,33}$ Other research indicates that persons with risk-taking tendencies or who engage in delinquent behaviors are more likely to enlist in the military. ${ }^{34}$ Both risk-taking and delinquent behavior have been linked to increased use of alcohol. ${ }^{35,36}$ This line of reasoning suggests that military service by itself may not lead to increased use of alcohol. Rather, individuals who join the military are otherwise more likely to use alcohol. That is, they would be more likely to use alcohol even if they did not enter the military. Accordingly, if this perspective is correct, military service should not be positively linked to alcohol use after accounting for risk-taking or delinquent behavior. We are able to examine this proposition because we use longitudinal data that follow men and women over time, including periods of military service, as well as measures of risk-taking and delinquent behavior.

\section{Data and Methods}

We use data taken from the NLSY-97. Starting in 1997, the NLSY-97 interviewed 8,984 respondents between the ages of fourteen and sixteen who were born between 1980 and 1984. Follow-up interviews in the NLSY-97 are conducted annually. The young men and women in our sample were eligible to be observed up to fourteen times over the period 1997-2010 with a retention rate of 83.2 percent as of 2010. The NLSY-97 data are representative of the US population born between 1980 and $1984 .^{37}$

Our regression analysis is based on person years where respondents contribute a person year for each round of the NLSY-97 in which they were interviewed. ${ }^{38}$ In other words, respondents contribute person years until they are lost to follow-up or they reach the last wave of the study (2010). For example, a respondent who was interviewed in all fourteen years of the interview would be represented fourteen 
times. The final sample includes information that follows men and women between the ages of fourteen and thirty, or the oldest age at which they continue to be interviewed. Person years follow the logic of life-course trajectories as they unfold over time. For example, respondents are followed as they move from civilian life, to being in the military, to being a veteran. Thus, we can examine changes in alcohol consumption as they occur at the same time as there are changes in military service. If all 8,984 original respondents had remained in the survey, there would be 125,776 person years in our data. Because we lose information on respondents who are lost to follow-up, our pooled sample includes 108,602 person years.

Our dependent variable is a time-varying measure of alcohol consumption measured each year in the NLSY-97. Thus, this measure can take a different value for each year a respondent is represented in the data, allowing us to model change in alcohol consumption over time. Four questions were asked of each respondent: "During the last 30 days, on how many days did you have one or more drinks of an alcoholic beverage?," "In the past 30 days, on the days that you drank alcohol, about how many drinks did you usually have?," "On how many days did you have five or more drinks on the same occasion during the past 30 days? By occasion we mean at the same time or within hours of each other?," "In the last 30 days, how many days have you had something alcoholic to drink such as beer, wine, or hard liquor right before or during school or work hours?" For each variable, respondents were assigned a value of $0,1,2$, or 3 for each question, corresponding to which quartile the respondent scored on each question ( 0 if the respondent scored in the first quartile, 1 if the respondent scored in the second quartile, 2 if the respondent scored in the third quartile, and 3 if the respondent scored in the fourth quartile). The resulting values for these quartile scores were then summed across the four questions. The result is a score for each of the fourteen years that ranges from 0 to 12 with higher scores indicating greater alcohol use.

We make use of two primary time-varying independent variables. The first variable is a dichotomous indicator of whether the respondent is currently serving on active duty in the military ( 409 men and 106 women at some point over the fourteen years). The second variable is a dichotomous indicator of whether the respondent is currently a veteran of active duty military service (261 men and 79 women at some point over the fourteen years; the difference between number of enlistees and number of veterans reflects the fact that a number of men and women remained in the military as of 2010). We also make use of a time-varying indicator of cumulative time spent on active duty in the military for respondents serving, measured in weeks. Finally, because the period covered by the NLSY-97 data includes the wars in Afghanistan and Iraq, we include a dichotomous indicator of whether the respondent reported having ever served in a combat zone (Did you ever serve in a combat or war zone?). Unfortunately, the question on service in a combat zone was only asked in 2009 and 2010 and does not indicate when or for how long combat service occurred (thus, it is not a time-varying covariate). Service in a combat zone, however, could have occurred at any point in time during a respondent's active duty service and is 
not restricted to the 2009-2010 period. Moreover, the question refers to service in a combat zone and does not determine where or whether respondents actually experienced combat. Overall, 179 men reported having served in a combat zone, but only twenty-one women.

We make use of an extended list of time-varying control variables in order to minimize the risk of obtaining spurious results linked to selectivity into the military. The time-varying covariates we control for include a dummy variable indicating that the respondent is currently enrolled in school; the number of weeks the respondent was employed in the past year; two dummy variables indicating whether the respondent was either cohabiting or married; the number of years of schooling obtained; a five-level measure of the respondent's self-evaluated health $(1=$ excellent, $2=$ very good, 3 =good, $4=$ fair, $5=$ poor); a continuous measure indicating the ratio of household income to the poverty level in each year; the number of children the respondent has; and age measured in months.

In addition to these demographic controls, we implement a time-varying control measuring the degree of delinquent behavior (risk taking) in which the respondent has engaged. The measure of delinquency consists of a time-varying, cumulative delinquency index that ranges from zero to ten, with higher scores indicating a greater degree of delinquency. The score was constructed by summing the responses to a set of ten questions ascertaining the number of delinquent/criminal acts in which respondents have ever engaged. ${ }^{39}$ A change in a respondent's score indicates that in a particular year a respondent engaged in a delinquent behavior that was not listed in earlier intervals. A value of 0 indicates that the respondent has not yet engaged in any of the activities listed. A value of 10 indicates that the respondent has engaged in each of the ten items listed at least once. Weighted scores were assigned to respondents who answered at least eight or more of the ten delinquency questions listed subsequently. ${ }^{40}$ Note that the index is based on the self-reports and does not necessarily reflect official records of delinquent behavior for an individual. The delinquency index has been used by other researchers to describe patterns of delinquency across the life course of adolescents ${ }^{41-43}$ and has considerable construct validity. The delinquency index is highly related to both enlistment in the military and alcohol consumption ${ }^{44}$ and can be construed to be a time-varying measure of a respondent's level of risk taking. ${ }^{45}$

Descriptive statistics for the pooled sample of yearly intervals are shown in Table 1. The mean level of the alcohol use index across the fourteen intervals is 3.62 based on an index that runs from 0 to 12 . About 2 percent of the intervals $(2,172$ intervals) include a respondent enlisted in the military, and about 1.5 percent of the intervals (1,692 intervals) include a veteran. Across intervals that include respondents with military service, the mean number of weeks served is 151.68 (2.92 years). The mean level of the delinquency index across intervals is 2.29 based on an index that runs from 0 to 10 . The mean age of respondents in months is 261.02 (21.75 years). For the other control variables, the means are 11.74 for years of schooling obtained, 2.10 for general health, 3.29 for the income to poverty level ratio, .42 for number 
Table I. Descriptive Statistics for Time-varying Variables Used in the Analysis of Alcohol Use. NLSY-97.

\begin{tabular}{lrrr}
\hline Variable & Mean & SD & Range \\
\hline Alcohol use & 3.62 & 3.88 & $0-12$ \\
Enlisted & 2.17 & & $0-1$ \\
Veteran & 1.54 & & $0-1$ \\
Age in months & 261.07 & 51.21 & $154-377$ \\
Weeks of service (among veterans and enlistees) & 151.68 & 117.15 & $1-644$ \\
Delinquency index & 2.29 & 2.42 & $0-10$ \\
Number of children & 0.42 & 0.84 & $0-9$ \\
Poverty ratio & 3.29 & 3.49 & $0-32.27$ \\
General health & 2.10 & 0.95 & $1-5$ \\
Education in years & 11.74 & 2.76 & $0-20$ \\
Married & 13.72 & & $0-1$ \\
Cohabiting & 11.97 & & $0-1$ \\
Weeks worked in the past year & 29.94 & 21.52 & $0-52$ \\
Enrolled in school & 43.94 & & $0-1$ \\
\hline
\end{tabular}

Note: $N=108,602$ intervals.

of children, 14 for the percent of intervals involving a married respondent and 12 for the percent of intervals involving a cohabiting respondent, 29.94 for the number of weeks worked in the past year, and 44 for the percent of intervals involving a respondent enrolled in school.

\section{Statistical Model}

We use a fixed-effects regression model to examine the relationship between the covariates and alcohol use. ${ }^{46}$ The fixed-effects procedure focuses on changes in alcohol use over time, and it automatically controls for all measured and unmeasured stable person-specific factors (e.g., race, parental background, and stable personality characteristics) that may act as fixed sources of spuriousness associated with entry into the military and alcohol use. (Stable person-specific factors are subtracted out when examining change in variables across time). Transitory sources of spuriousness (those associated with unobserved changes in characteristics linked to military service) are not controlled in fixed-effects models. Yet the model does include a number of important time-varying characteristics of individuals that should help alleviate this issue (i.e., delinquency, marital status, education, income, school enrollment, children, and health).

\section{Results}

Multivariate results are shown in Table $2 .{ }^{47}$ Three models are presented. Each model is based on a fixed-effects regression that controls for all fixed covariates such as 
Table 2. Fixed-effects Regression Results for the Analysis of Alcohol Use (Alcohol Index): NLSY-97.

\begin{tabular}{lrrr}
\hline \multirow{2}{*}{ Variable } & \multicolumn{3}{c}{ Fixed effects estimates } \\
\cline { 2 - 4 } & Model I & Model 2 & Model 3 \\
\hline Enlisted (for women when male $=0$ ) & $-0.637^{* *}$ & $-0.750^{* *}$ & $-0.484^{* *}$ \\
Veteran (for women when male $=0$ ) & $-0.591^{* *}$ & $-0.621^{* *}$ & $-0.65 I^{* *}$ \\
Male $\times$ enlisted & $1.187^{* *}$ & $1.099^{* *}$ & $1.169^{* *}$ \\
Male $\times$ vet & $0.914^{* *}$ & $0.916^{* *}$ & $0.996^{* *}$ \\
Age in months & $0.124^{* *}$ & $0.124^{* *}$ & $0.058^{* *}$ \\
Age Squared $\times$ I,000 & $-0.212^{* *}$ & $-0.212^{* *}$ & $-0.100^{* *}$ \\
Length of service in weeks & $0.002^{* *}$ & $0.002^{* *}$ & $0.003^{* *}$ \\
Enlisted $\times$ combat & & $0.505^{* *}$ & $0.510^{* *}$ \\
Veteran $\times$ combat & & 0.086 & -0.061 \\
Delinquency index & & & $0.242^{* *}$ \\
Number of children & & & $-0.179^{* *}$ \\
Poverty ratio & & & $0.007^{* *}$ \\
General health & & & $0.163^{* *}$ \\
Education in years & & & $0.241^{* *}$ \\
Married & & & $-0.744^{* *}$ \\
Cohabiting & & & $-0.440^{* *}$ \\
Weeks worked in the past year & & $0.010^{* *}$ \\
Enrolled in school & & & $0.124^{* *}$ \\
\hline
\end{tabular}

Note: The effect of enlistment for women is $-.637+1.187(0)=-.637$. The effect of enlistment for men is $-.637+\mathrm{I} .187(\mathrm{I})=.550$. The effect of being a veteran for women is $-.59 \mathrm{I}+.914(0)=-.59 \mathrm{I}$. The effect of being a veteran for men is $-.591+.914(\mathrm{I})=.323 . \mathrm{N}=108,602$ intervals. $* * p<.05$.

race, stable personality characteristics, parental background, and so on. Model 1 includes the effects of the measures of military service (enlisted, veteran, and length of service), as well as a simple control for age. The model also includes an interaction between gender (here, male takes the value of 1) and the variables indicating whether the interval involves either a service member or a veteran. Although fixed characteristics (e.g., gender) cannot be included in a fixed-effects model, interactions between fixed characteristics and time-varying characteristics can be included. Thus, the interaction between male and enlisted, in conjunction with the coefficient for enlistment, allows us to determine how the effect of enlistment varies according to gender. Similarly, the interaction between male and veteran, in conjunction with the coefficient for being a veteran, allows us to determine how the effect of being a veteran varies according to gender. Each of the coefficients in the model is statistically significant at conventional levels. If selection into the military was based on a proclivity to use alcohol, the fixed-effects estimates for military service would not be statistically significant from zero. That is, there would be no change in alcohol use associated with military service. However, that is not the case, thus showing support for a direct effect of military service on alcohol use. 
The coefficient for enlistment indicates that women who enlist in the military are less likely to use alcohol than comparable female respondents who do not enlist. The difference is -.637 units on the alcohol use index. For men who enter the military, however, there is an increase in alcohol use. The increase is $(1.187-.637=) .550$ on the alcohol use index. For veteran status, a similar pattern emerges. Female veterans score .591 units lower on the alcohol use index, whereas male veterans score $(.914-.591=) .323$ units higher on the alcohol index. In addition, duration of military service is linked to increased alcohol use, about $(.002 \times 52=) .10$ units on the alcohol use index for each additional year of service. Thus, a male respondent with one year of service would score $.56(=.550+.1 \times 1)$ higher on the alcohol index, and a male respondent with five years of service would score $.6(=.550+.1 \times 5)$ units higher. Finally, the coefficients for age indicate an increase in alcohol use as respondents age, but at a decreasing rate at older ages (the linear term, .124 is positive, and the quadratic term, -.212 , is negative).

Model 2 adds combat service to model 1 in order to determine whether combat service may be the source of the relationship between military service and alcohol use. Recall that combat service as measured in the NLSY-97 is a fixed characteristic and can only be included as part of an interaction with a time-varying variable. Model 2 allows combat service to interact with enlistment status and being a veteran. The results indicate that combat service does not affect alcohol use for veterans, but increases alcohol use for enlisted service members by .505 on the alcohol use index. Moreover, the effects of enlistment and veteran status are generally similar to those shown in model 1 , indicating that service in a combat zone does not explain the effects of military service on alcohol use, although it further increases use.

Model 3 adds a number of time-varying control variables to model 2, including the delinquency index, as a further search for covariates that can explain the relationship between military service and alcohol use. The addition of the covariates does little to alter the effects of military service on alcohol use, indicating that the effects of serving cannot be attributed to these time-varying covariates. Women enlisted in the military score .484 lower on the alcohol use index, compared to a score $(1.169-.484=) .685$ higher for men enlisted in the military. For veterans, women score .651 lower on the alcohol use index, compared to a score that is $(.996-.651=)$ .345 higher for men. Being a veteran with combat service does not alter alcohol use. However, being enlisted combined with having been in a combat zone increases alcohol use by .510 units. Additional years of service lead to an increase in the alcohol use index by $(.003 \times 52=) .156$.

\section{Discussion}

The results are generally in line with our expectations. In the United States, male enlistees and veterans are more likely than their civilian counterparts to use alcohol. The opposite is true for female enlistees and veterans. The longer someone serves, the more likely they are to use alcohol, although the effect is small. Enlistees who 
have served in a combat zone are the most likely to use alcohol, but there is no relationship between being a veteran, having served in a combat zone and alcohol use.

Our results are the first to link military service to use of alcohol using longitudinal data that allow comparisons between civilians and those who have served. The longitudinal data provide multiple observations on each individual, allowing us to employ a fixed-effects model that provides a degree of control over unmeasured selectivity into the military. Our results are free of any selectivity that is tied to time constant variables, either measured or unmeasured. Of course, it is still possible that time-varying factors may be linked to selectivity into the military, but we employed a large set of important time-varying demographic characteristics that may be linked to military service and alcohol use. We were also able to control for a time-varying indicator of risky behavior (delinquency) that prior research has found to be related to military service and alcohol use. The results, therefore, imply that the relationship between military service and alcohol use is not likely a function of selectivity.

Although our results are in line with expectations, a couple of findings are worthy of additional consideration. First, women who serve in the military are not only less likely to use alcohol than men who serve in the military, but they are less likely to use alcohol than their civilian counterparts. This implies that there is something about military service that reduces alcohol consumption for women. It is not immediately evident why this negative relationship should occur. Obviously, women react differently to their experience in the military than do men. We suspect that part of the reason for the negative link between military service and alcohol use for women is the threat of sexual harassment and assault that is common in the military. ${ }^{48}$ Alcohol use is tightly linked to sexual assault, both within and outside the military, and women who serve may become particularly aware of this linkage. It may also be the case that in order to justify their place in the military that women abstain from using alcohol, especially to the extent that their participation in particular military occupation specialties based on use of alcohol is subject to critical review based on their gender.

A second finding worth commenting upon is the fact that having served in a combat zone increases alcohol use for currently enlisted service members but not for veterans. As was the case for reduced alcohol use among women service members, it is not immediately clear why this difference would occur. One possibility may be tied to the fact that currently enlisted respondents have served longer than have veterans (results not shown). Accordingly, it is possible that currently enlisted respondents have experienced more than one tour of duty in a combat zone and may suffer from stress-induced alcohol use associated with the prospect of another deployment to a combat zone, something that cannot be determined with our data. It is also possible that reenlistment in the military (which is more likely to have occurred for currently enlisted service members) represents an unmeasured and time-varying source of selectivity positively linking military service to alcohol use. It may also be the case that any relationship between veteran status and alcohol use may occur only later in life (here later than age thirty). 
Despite its strengths, our analysis also suffers from a number of weaknesses. One weakness is that despite the longitudinal nature of the data, the number of respondents who have served in the military is relatively small, especially for women. The small number of respondents in our sample who served in the military means that we are not able to make use of more detailed information about military service such as branch of service, rank, and military occupation. At best, we have provided estimates of the effects of military service that likely hide variation in use that occurs according to characteristics of service. Moreover, the relatively small number of service members and veterans relative to nonveterans makes it difficult to make more refined comparisons. A second weakness of the NLSY-97 data is that the measure of combat service does not provide any information about actual exposure to combat or its timing. As such, we suspect that the effect of combat on alcohol use may be underestimated in our models. The results of our analysis are also limited to a particular piece of historical time, roughly the first decade of the new millennium. We are not able to draw any conclusions about the relationship between military service and alcohol use during any other period. A third weakness is that the NLSY-97 data only follow respondents until they are about age thirty. This limitation is particularly important for considering the effects of veteran status on alcohol use. We did not find evidence that the effect of being a veteran declined with time, but this could change as veterans in the NLSY-97 continue to age. Most of the veterans in our data are relatively recent veterans with most of their life course still ahead of them. Finally, our results are specific to the United States, and military service in other contexts may impact alcohol use in a different fashion, if at all.

\section{Conclusion}

Despite its limitations, our study is the first to use longitudinal data and a fixedeffects approach to provide evidence that military service affects alcohol use and in a different direction for men and women. The culture of alcohol use in a highly age-stratified environment appears to encourage men to consume more alcohol. It is also the case that the effect of military service is not limited to the time that men spend in the military. Male veterans are also more likely to consume alcohol than are comparable civilians. Although we expected that women experiencing military service to drink less than their male counterparts, we found that women who serve are less likely to drink than their civilian counterparts.

The results that we present are important for policy makers to consider. Prior research has indicated that military service, both current service and being a veteran, is positively linked to alcohol consumption. What has been missing, however, is evidence that military service may play a causal role in alcohol use. Cross-sectional results are subject to the possibility that selection leads to the positive relationship between military service and alcohol use. Our models, while not perfect, provide evidence that military service leads to more alcohol consumption among service members that would have been the case if they had not served. This finding should 
provide for increased emphasis on efforts to reduce the culture of alcohol consumption in the military. The results should also spur more investigation into the unique role that gender plays in the linkage between military service and alcohol use.

\section{Declaration of Conflicting Interests}

The authors declared no potential conflicts of interest with respect to the research, authorship, and/or publication of this article.

\section{Funding}

The authors disclosed receipt of the following financial support for the research, authorship, and/or publication of this article: This research was supported by grant no. R15 HD069958 from the National Institute of Child Health and Human Development. Any opinions, findings, and conclusions expressed in this material are those of the author and do not necessarily reflect the views of NICHD.

\section{Notes}

1. Institute of Medicine, Substance Use Disorders in the U.S. Armed Forces (Washington, DC: Institute of Medicine, The National Academies Press, 2012).

2. Substance Abuse and Mental Health Services Administration, "Alcohol Use among Veterans," The NHSDA Report, accessed August 1, 2013, http://www.samhsa.gov/ data/2k2/NSvets/NSvets.htm.

3. Institute of Medicine, Substance Use Disorders in the U.S. Armed Forces, 2012.

4. Hoerster et al., "Health and Health Behavior Differences: U.S. Military, Veteran, and Civilian Men," American Journal of Preventative Medicine 43, 5 (2012): 483-89.

5. Institute of Medicine, Substance Use Disorders in the U.S. Armed Forces, 2012.

6. Wyatt Olson, "Military Brass Dealing with an Entrenched Culture of Alcohol in Asia," Stars and Stripes, December 10, 2012.

7. Bohnert et al., "Veteran Status and Alcohol Use in Men in the United States," Military Medicine 177, 2 (2012): 198-203.

8. Hoerster et al., Health and Health Behavior Differences, 2012

9. Substance Abuse and Mental Health Services Administration, "Alcohol Use among Veterans," 2013.

10. Bohnert et al., "Veteran Status and Alcohol Use in Men in the United States," 2012.

11. Ibid.

12. Bray et al., "Regional Differences in Alcohol Use among U.S. Military Personnel," Journal of Studies on Alcohol 66, 2 (2005): 229-38.

13. Bray et al., "Substance Use and Mental Health Trends among U.S. Military Active Duty Personnel: Key Findings from the 2008 DoD Health Behavior Survey," Military Medicine 175, 6 (2010): 390-99.

14. Mattiko et al., "Alcohol Use and Negative Consequences among Active Duty Military Personnel," Addictive Behaviors 36, 6 (2011): 608-14.

15. Meade Eggleston, Kristy Straits-Tröster, and Harold Kudler, "Substance Use Treatment Needs among Recent Veterans,” North Carolina Medical Journal 70, 1 (2009): 54-58. 
16. Amy Wallace, Atticus Wallace, and William Weeks, "The U.S. Military as a Natural Experiment: Changes in Drinking Age, Military Environment, and Later Alcohol Treatment Episodes among Veterans," Military Medicine 173, 7 (2008): 619-25.

17. Yu-Chu Shen, Jeremy Arkes, and Thomas Williams, "Effects of Iraq/Afghanistan Deployments on Major Depression and Substance Use Disorder: Analysis of Active Duty Personnel in the US Military," American Journal of Public Health 102, S1 (2012): S80-87.

18. Genevieve Ames, Carol Cunradi, and Roland Moore, "Alcohol, Tobacco, and Drug Use among Young Adults Prior to Entering the Military," Prevention Science 3, 2 (2002): 135-44.

19. Bohnert et al., "Veteran Status and Alcohol Use in Men in the United States," 2012.

20. Bray et al., "Substance Use and Mental Health Trends among U.S. Military Active Duty Personnel," 2010.

21. Eggleston, Straits-Tröster, and Kudler, "Substance Use Treatment Needs among Recent Veterans among Recent Veterans,” 2009.

22. John Donovan, "Adolescent Alcohol Initiation: A Review of Psychosocial Risk Factors," Journal of Adolescent Health 35, 6 (2005): 529.e7-18.

23. Marion Mundt, "The Impact of Peer Social Networks on Adolescent Alcohol Use Initiation," Academic Pediatrics 11, 5 (2011): 414-21.

24. Olson, "Military Brass Dealing with and Entrenched Culture of Alcohol in Asia," 2012.

25. Jane Kroger, Identity Development: Adolescence through Adulthood (Thousand Oaks, CA: Sage, 1999).

26. Ralph Hingson, Timothy Heeren, and Michael Winter, "Age at Drinking Onset and Alcohol Dependence," Archives of Pediatrics and Adolescent Medicine 160, 7 (2006): 739-46.

27. Isabel Jacobson et al., "Alcohol Use and Alcohol-related Problems Before and After Military Combat Deployment," Journal of the American Medical Association 300, 6 (2008): 663-75.

28. Susan Eisen et al., "Mental and Physical Health Status and Alcohol and Drug Use Following Return from Deployment to Iraq or Afghanistan," American Journal of Public Health 102, S1 (2012): S66-S73.

29. Keren Eisen, "Health Indicators for Military, Veteran, and Civilian Women," American Journal of Preventative Medicine 42, 5 (2012): 473-80.

30. Amy Wallace, Edward Sheehan, and Yinong Young-Xu, "Women, Alcohol, and the Military: Cultural Changes and Reductions in Later Alcohol Problems among Female Veterans," Journal of Women's Health 18, 9 (2009): 1347-53.

31. Susan Nolen-Hoeksema, "Gender Differences in Risk Factors and Consequences for Alcohol Use and Problems," Clinical Psychology Review 24, 8 (2009): 981-1010.

32. Ames, Cunradi, and Moore, "Alcohol, Tobacco, and Drug Use among Young Adults Prior to Entering the Military," 2002.

33. Adam Barry et al., "Examining the Association between Binge Drinking and Propensity to Join the Military," Military Medicine 178, 1 (2013): 37-42.

34. Sylvia Young et al., "Risky Alcohol Use, Age at Onset of Drinking, and Adverse Childhood Experiences in Young Men Entering the US Marine Corps," Archives of Pediatrics and Adolescent Medicine 160, 12 (2006): 1207-14. 
35. NLSY97 Users Guide, accessed March 11, 2013, http://www.bls.gov/nls/handbook/2005/ nlshc2.pdf.

36. In essence, by using person years, differences in alcohol use from year to the next can be regressed on differences in the covariates from one year to the next. Estimates are therefore based on within-person variation and not between-person variation. Because differences are used, the effects of all stable characteristics of respondents, either measured or unmeasured, are removed from the estimates via subtraction. More detail on fixed-effects estimates is provided by Paul Allison, "Using Panel Data to Estimate the Effects of Events," Sociological Methods and Research 23, 2 (1994): 174-99.

37. See note 35 .

38. See note 36 .

39. The questions used to form the Delinquency Index are as follows:

- Have you ever run away, that is, left home and stayed away at least overnight without your parent's prior knowledge or permission?

- Have you ever carried a hand gun? When we say hand gun, we mean any firearm other than a rifle or shotgun.

- Have you ever belonged to a gang?

- Have you ever purposely damaged or destroyed property that did not belong to you?

- Have you ever stolen something from a store or something that did not belong to you worth less than fifty dollars?

- Have you ever stolen something from a store, person, or house, or something that did not belong to you worth fifty dollars or more including stealing a car?

- Have you ever committed other property crimes such as fencing, receiving, possessing or selling stolen property, or cheated someone by selling them something that was worthless or worth much less than what you said it was?

- Have you ever attacked someone with the idea of seriously hurting them or have a situation end up in a serious fight or assault of some kind?

- Have you ever sold or helped sell marijuana (pot, grass), hashish (hash), or other hard drugs such as heroin, cocaine, or LSD?

- Have you ever been arrested by the police or taken into custody for an illegal or delinquent offense (do not include arrests for minor traffic violations)?

40. That is, respondents who answered at least eight of the ten questions were given a score that was adjusted to a base of ten. For example, if a respondent answered positively to four of the eight questions they were assigned a value of five for the scale $(4 / 8 \times 10)$. Respondents who answered fewer than eight items were assigned a missing value for the scale and received an imputed value using the chained equations approach described previously.

41. Cory Bolken, “Early Adolescents' Perceptions of Mothers' and Fathers' Parenting Styles and Problem Behavior," Marriage and Family Review 46, 8 (2010): 563-79.

42. Elizabeth Hair et al., "Risky Behavior in Late Adolescence: Co-occurrence, Predictors, and Consequences," Journal of Adolescent Health 45, 3 (2009): 253-61. 
43. Erin Holmes, Hinckley Jones-Sanpei, and Randal Day, "Adolescent Outcome Measures in the NLSY97 Family Process Data Set: Variation by Race and Socioeconomic Conditions," Marriage and Family Review 45, 4 (2009): 374-91.

44. Jay Teachman and Lucky Tedrow, "Delinquent Behavior, the Transition to Adulthood, and the Likelihood of Military Enlistment," Social Science Research 45, 2 (2014): 46-55.

45. The NLSY-97, like any other large, longitudinal study is not immune to problems associated with missing data. To deal with this issue, we used a chained equation approach to imputing missing data available in STATA. All results reported in this article are based on five imputed databases using STATA's multiple imputation procedure. The dependent variable (alcohol use index) was used in the imputation procedure; however, all cases with missing data on the alcohol index were dropped from the analysis after missing values were imputed for all other variables.

46. The fixed-effects model we estimate is of the following general form:

$\mathrm{ALCINDEX}_{i t}=u_{1} \mathrm{Age}_{i t}+u_{2} \mathrm{Age}_{i t}^{2}+\delta_{1} \mathrm{ENLIST}_{i t}+\delta_{2} \mathrm{VET}_{i t}+\delta_{3} \mathrm{DURSERVICE}_{i t}+\gamma W_{i t}+\alpha_{i}+\varepsilon_{i t}$,

where ALCINDEX $_{i t}$ represents an individual $i$ 's score on the alcohol use index at time $t$. The fourteen possible values of $t$ correspond to the fourteen survey rounds covered in our analysis. The value of $i$ for a given $t$ depends upon the number of respondents who contribute an observation at that value of $t$. Age ${ }_{i t}$ indicates the age of the respondent at time $t$ (and is used to account for change in alcohol use associated with aging); ENLIST $i t$ represents a time-varying dummy variable indicating whether respondent $i$ is enlisted in the military at time $t ; \mathrm{VET}_{i t}$ is a time-varying dummy variable indicating whether respondent $i$ is a veteran at time $t$; DURSERVICE ${ }_{i t}$ is a time-varying indicator of the length of time spent in the military at time $t$ for enlistees and veterans; $W_{i t}$ represents a vector of timevarying characteristics of respondents, both nonveterans and veterans, that correspond to control variables; $u_{1}, u_{2}, \delta_{1}-\delta_{3}$, and $\gamma$ are coefficients or vectors of coefficients, $\alpha_{i}$ represents unobserved and constant person-specific differences across respondents that affect alcohol use, and $\varepsilon_{i t}$ is a residual error term. This model is estimated using XTREG in STATA with a fixed-effects option.

The model does not include any fixed (nonchanging) characteristics because in the fixedeffects procedure the effects of any constant person-specific characteristics such as race or stable personality characteristics are absorbed within the person-specific factors, $\alpha_{i}$. Thus, although covariates such as race do not appear in the model, their effects on the dependent variable are controlled (as noted later, however, interactions between fixed characteristics and time-varying characteristics can be included in the model). Heuristically, one can imagine that the fixed-effects model is estimated by including a set of dummy variables corresponding to each of the respondents.

47. We note two extensions of the multivariate results shown in Table 2 (results not shown). First, we sought to determine whether the relationship between veteran status and alcohol use depends on how long a respondent has been a veteran. Our thought was that the effect of being a veteran decays over time with less exposure to military life. When we included a control for duration of time since becoming a veteran, the coefficient was not 
statistically significant, and the effects of the other variables were unchanged.

Second, we sought to determine whether the military variables would be related not just to overall alcohol use but to what can be construed to be alcohol abuse. To accomplish this task, we first determined the relationship between the alcohol use index and one of its component parts - the number of days in the past thirty days that a respondent has had five or more drinks, which is a commonly used indicator of problematic alcohol use. We determined that after a score of four on the alcohol use index, about 70 percent of respondents scored in the top quartile of individuals having five or more drinks in a day. Over 90 percent of these respondents scored in the top two quartiles of individuals having five or more drinks in a day. Accordingly, we replicated the models shown in Table 2 using a dichotomous indicator of whether the respondent scored five or higher on the alcohol use index. The results mirror those shown in Table 2. That is, women who are enlisted or who are veterans are less likely to abuse alcohol. Men who are enlisted or who are veterans are more likely to abuse alcohol, with the effect being stronger for men currently enlisted. In addition, duration of service was positively related to alcohol abuse, as was being an enlisted person having served in a combat zone.

48. US Department of Defense, Annual Report on Sexual Assault, 2012, accessed August 16, 2013, http://www.sapr.mil/public/docs/reports/FY12_DoD_SAPRO_Annual_Report_on _Sexual_Assault-VOLUME_ONE.pdf.

\section{Author Biography}

Jay Teachman is currently a professor in the Department of Sociology at Western Washington University. His research interests focus on the relationship between military service and subsequent life-course outcomes for men and women. Recent publications include M. Whyman, M. Lemmon, and J. Teachman. 2011. "Non-Combat Military Service in the United States and its Effects on Depressive Symptoms among Men." Social Science Research 40:695-703; Teachman, Jay. 2010. "Are Veterans Healthier? Military Service and Health at Age 40 in the All-volunteer Era." Social Science Research 40:326-35; J. Teachman and L. Tedrow. 2008. "Divorce, Race, and Military Service: More than Equal Pay and Equal Opportunity." Journal of Marriage and Family 70:1030-44.

Carter Anderson was an undergraduate student at Western Washington University and an undergraduate student research assistant on the NICHD grant that funded this research. In the Fall 2014 she will be a graduate student in the Department of Sociology at the University of Washington. Her main research interests are gender and stratification. She has a forthcoming book chapter, "Demographic Changes: Age at First Marriage" in L. Ganong, M. Coleman, and J. Golson, The Social History of the American Family co-authored by J. Teachman, C. Anderson, and L. Tedrow.

Lucky M. Tedrow is the Director, Demographic Research Laboratory, Center for Social Science Instruction, Western Washington University. His research areas span applied, social and formal demography. Research interests include antecedents and 
outcomes of military service. Computer applications and undergraduate instruction are main areas of focus. He has been the PI on seven NSF funded grants for undergraduate education. Recent publications include J. Teachman and L. Tedrow. 2014. "Delinquent Behavior, the Transition to Adulthood, and the Likelihood of Military Enlistment." Social Science Research 45:46-55; J. Teachman and L. Tedrow. 2013. "Veteran Status and Body Weight: A Longitudinal Fixed-effects Approach." Population Research and Policy Review 32:199-220; D. Swanson and L. Tedrow. 2012. "Using Cohort Change Ratios to Estimate Life Expectancy in Populations with Negligible Migration: A New Approach." Canadian Studies in Population 39:83-90. 